\title{
A!
}

This is an electronic reprint of the original article.

This reprint may differ from the original in pagination and typographic detail.

Heinonen, Juha; Haarahiltunen, Antti; Serue, Michael; Vähänissi, Ville; Pasanen, Toni; Savin, Hele; Werner, Lutz; Juntunen, Mikko

High-sensitivity NIR photodiodes using black silicon

Published in:

SPIE Conference Proceedings

DOI:

$10.1117 / 12.2544756$

Published: 01/01/2020

Document Version

Publisher's PDF, also known as Version of record

Please cite the original version:

Heinonen, J., Haarahiltunen, A., Serue, M., Vähänissi, V., Pasanen, T., Savin, H., Werner, L., \& Juntunen, M. (2020). High-sensitivity NIR photodiodes using black silicon. SPIE Conference Proceedings, 11276, [112760G]. https://doi.org/10.1117/12.2544756

This material is protected by copyright and other intellectual property rights, and duplication or sale of all or part of any of the repository collections is not permitted, except that material may be duplicated by you for your research use or educational purposes in electronic or print form. You must obtain permission for any other use. Electronic or print copies may not be offered, whether for sale or otherwise to anyone who is not an authorised user. 


\section{High-sensitivity NIR photodiodes using black silicon}

Heinonen, Juha, Haarahiltunen, Antti, Serue, Michael Dov, Vähänissi, Ville, Pasanen, Toni, et al.

Juha Heinonen, Antti Haarahiltunen, Michael Dov Serue, Ville Vähänissi, Toni P. Pasanen, Hele Savin, Lutz Werner, Mikko A. Juntunen, "High-sensitivity NIR photodiodes using black silicon," Proc. SPIE 11276, Optical Components and Materials XVII, 112760G (3 March 2020); doi: 10.1117/12.2544756

SPIE. Event: SPIE OPTO, 2020, San Francisco, California, United States 


\title{
High-sensitivity NIR photodiodes using black silicon \\ Juha Heinonen $^{\mathrm{a}}$, Antti Haarahiltunen ${ }^{\mathrm{a}}$, Michael Dov Serue ${ }^{\mathrm{a}}$, Ville Vähänissi ${ }^{\mathrm{b}}$, Toni P. Pasanen ${ }^{\mathrm{b}}$, Hele Savin $^{\mathrm{b}}$, Lutz Werner ${ }^{\mathrm{c}}$, and Mikko A. Juntunen ${ }^{\mathrm{a}}$ \\ ${ }^{a}$ ElFys, Inc., Tekniikantie 12, 02150 Espoo, Finland; ${ }^{\mathrm{b}}$ Department of Electronics and \\ Nanoengineering, Aalto University, Tietotie 3, 02150 Espoo, Finland; ${ }^{c}$ Physikalisch-Technische \\ Bundesanstalt, Abbestr. 2-12, 10587 Berlin, Germany
}

\begin{abstract}
There is an increasing demand for highly sensitive near infrared (NIR) detectors due to many rapidly growing application areas, such as LiDAR and optical communications. Despite the limited NIR absorption, silicon is a common substrate material in NIR detectors due to low cost and maturity of the technology. To boost the NIR performance of silicon devices, one option is texturizing the front and/or back surface to reduce reflectance and extend the optical path by scattering. Here we demonstrate silicon photodiodes with nanostructured front surface (i.e. black silicon fabricated with reactive ion etching (RIE) that exhibit significantly enhanced external quantum efficiency (EQE) at NIR wavelengths compared to typical state-of-the-art silicon photodiodes. The detectors exhibit over 90\% EQE with wavelengths up to $1040 \mathrm{~nm}$ and above $60 \%$ at $1100 \mathrm{~nm}$. Identical detectors with a planar surface are also investigated revealing that the enhancement from black silicon effectively corresponds to increasing the substrate thickness up to $43 \%$ at $1100 \mathrm{~nm}$ depending on the thickness of the active layer and back surface structure. This confirms that in addition to reduced reflectance, scattering of transmitted light induced by black silicon plays a key role in the EQE enhancement which benefits especially devices such as backside illuminated photodetectors where very thin substrates are required. We also demonstrate that the high EQE of the black silicon detectors is maintained at incidence angles up to 60 degrees allowing excellent performance in applications where the light is not always perpendicular.
\end{abstract}

Keywords: Black silicon, near infrared, responsivity, silicon, photodiode

\section{INTRODUCTION}

There is an increasing demand for highly sensitive near infrared (NIR) detectors due to many rapidly growing application areas, such as LiDAR and optical communications. Despite the limited NIR absorption, silicon is a common substrate material in NIR detectors due to low cost and maturity of the technology. The silicon NIR performance can be boosted by increasing the length of the optical path. The most straightforward way is to increase the silicon wafer thickness, which is not the optimal solution in respect of cost and speed ${ }^{1-2}$. Better option is to utilize a light trapping structure and/or a texture that effectively scatters the light ${ }^{1-2}$. A light trapping structure maximizes internal reflectance from the front and back surface preventing light from leaving silicon. To maximize the amount of light that enters silicon and gets trapped, the external reflectance from the front surface needs to be minimized. Unfortunately, this often simultaneously results in reduced internal reflectance from the front surface for perpendicular light beam ${ }^{2}$. This can be averted if either the front or back (or both) surface scatters the light enhancing the probability of total internal reflection from the surfaces ${ }^{2-3}$.

Silicon photodiodes having nanostructured front surface (i.e. black silicon) fabricated with reactive ion etching (RIE) have almost prefect light response in a wide wavelength region from $200 \mathrm{~nm}$ to $1000 \mathrm{~nm}^{4-5}$. Black silicon (b-Si) eliminates virtually all reflections due to gradient refractive index and also simultaneously scatters light ${ }^{3,6}$ depending on the size of the nanostructures ${ }^{3}$. In this paper, we study b-Si photodiodes fabricated by inductively-coupled plasma reactive ion etching (ICP-RIE) and investigate the impact of light trapping provided by the nanostructures on external and internal quantum efficiency (EQE \& IQE) at NIR wavelengths.

Optical Components and Materials XVII, edited by Shibin Jiang, Michel J. F. Digonnet,

Proc. of SPIE Vol. 11276, 112760G · @ 2020 SPIE · CCC code: 0277-786X/20/\$21

doi: $10.1117 / 12.2544756$

Proc. of SPIE Vol. 11276 112760G-1 


\section{EXPERIMENTAL}

\subsection{Processing of devices}

The photodiodes were fabricated by ElFys, Inc., using process similar to the one previously described in ${ }^{4}$. Hence, only the main features are outlined here. Three different kind of n-type silicon starting wafers were used: $675 \mu \mathrm{m}$ thick double side polished (DSP) Float zone (FZ) wafers with $>10 \mathrm{k} \Omega \mathrm{cm}$ resistivity, $525 \mu \mathrm{m}$-thick single side polished (SSP) FZ wafers with $>10 \mathrm{k} \Omega \mathrm{cm}$ resistivity, and epitaxial wafers with $25 \mu \mathrm{m}$-thick $1 \mathrm{k} \Omega \mathrm{cm}$ epitaxial layer on top of $625 \mu \mathrm{m}$ thick $\sim 0.01$ $\Omega \mathrm{cm}$ carrier. The bulk minority-carrier lifetime in the $\mathrm{FZ}$ wafersis $>1 \mathrm{~ms}$ and the epitaxial layer has lifetime long enough so that it is not limiting charge collection. ICP-RIE was used to etch b-Si at cryogenic temperature. The b-Si was passivated using $20 \mathrm{~nm}$ thick atomic layer deposited (ALD) alumina $\left(\mathrm{Al}_{2} \mathrm{O}_{3}\right)$, which also forms an induced junction at the same time. Additionally, alumina was deposited on photodiodes without $\mathrm{b}$-Si to form planar induced junction photodiodes. The active area of the photodiodes was $5 \mathrm{~mm} \times 5 \mathrm{~mm}$ square. The induced junction region was surrounded by a boron-implanted area with aluminum on top to realize a p-type ohmic anode contact to the inversion layer. Similarly, the n-type cathode contact was fabricated by aluminum-covered phosphorus implantation on the backside.

\subsection{Measurements}

EQE of the b-Si photodetectors fabricated on a $525 \mu \mathrm{m}$ thick FZ wafer was measured by Physikalisch-Technische Bundesanstalt (PTB). The spectral responsivity of the detector was calibrated against several secondary detector standards at a measurement facility consisting of a double-grating monochromator with order-sorting filters and a detector positioning system. A xenon arc lamp and a tungsten halogen lamp were used as radiation sources in the wavelength ranges below and above $400 \mathrm{~nm}$, respectively. The photocurrents of the detector under test and of the secondary detector standards were measured in photovoltaic mode by using the same current-to-voltage converter and digital voltmeter. The spectral responsivity of the secondary detector standards had previously been calibrated against PTB's primary national detector standards for optical radiant power, the cryogenic electrical substitution radiometers. The relative standard measurement uncertainty of the b-Si photodetector EQE was between $0.1 \%$ and $0.4 \%$ in the UV range, $0.05 \%$ or smaller in the wavelength range between $420 \mathrm{~nm}$ and $960 \mathrm{~nm}$, and between $0.1 \%$ and $0.3 \%$ in the wavelength range between $980 \mathrm{~nm}$ and $1100 \mathrm{~nm}^{5}$

All the other EQE spectra were measured with a setup where the photodiode was illuminated with Bentham ILD-D2-QH24 dual-lamp light source. The lamp was coupled to Bentham TMC300_0060 monochromator where the wavelength was selected with $10 \mathrm{~nm}$ bandwidth and focused on the photodiode. The EQE values were initially calibrated against the Newport 818-UV photodetector and a photodiode measured by PTB was used as an additional reference in the NIR wavelengths. To obtain the internal quantum efficiencies (IQE) for absorption enhancement calculation, the external reflectance of the planar surfaces was calculated using transfer matrix method ${ }^{7}$ and values reported in ${ }^{4}$ were used for b-

$\mathrm{Si}$. The stage where the photodiode is mounted can be tilted which allowed the EQE measurements with different incidence angles. 


\section{RESULTS AND DISCUSSION}

\subsection{Spectral responsivity of black silicon photodiodes}

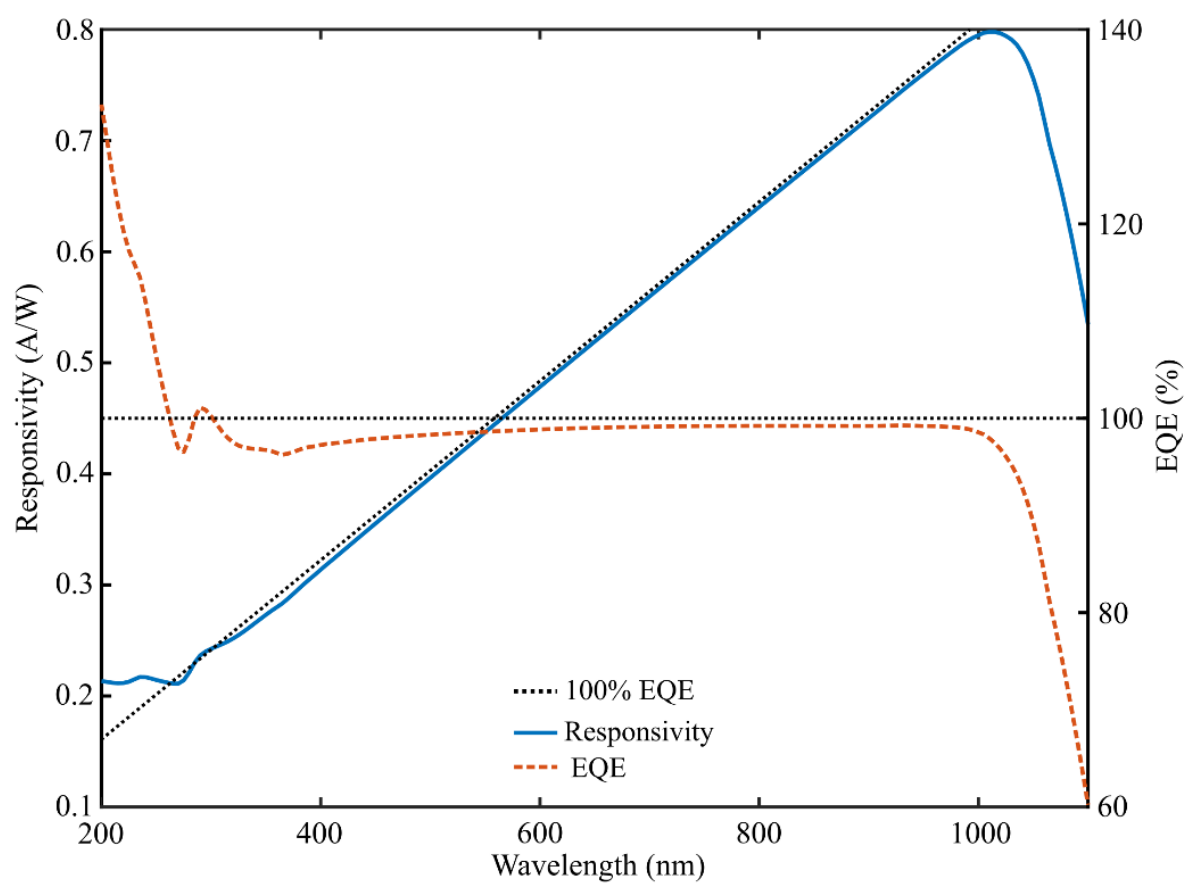

Figure 1. EQE and the corresponding responsivity measured by $\mathrm{PTB}^{5}$. The maximum EQE in VIS-NIR wavelengths is $99.2 \%$ and the maximum relative uncertainty of the measurement is $0.4 \%$. Curves representing ideal 100\% EQE are also shown as reference.

EQE and spectral response measured by PTB are shown in Figure 1. At ultraviolet (UV) wavelengths, the EQE exceeds $100 \%$ reaching the maximum of $132 \%$ at $200 \mathrm{~nm}$. A thorough discussion of the UV response can be found in ${ }^{5}$. Between $630 \mathrm{~nm}$ and $990 \mathrm{~nm}$, the EQE even exceeds $99 \%$. This clearly indicates that the b-Si in-homogeneities reported in ${ }^{8}$ can be avoided with careful process optimization. Furthermore, the EQE remains also exceptionally high at the NIR wavelengths $\left(84 \%\right.$ at $1060 \mathrm{~nm}$ ) compared to standard (typically <50\%) and NIR optimized silicon photodiodes $(\sim 72 \%)^{1}$. The excellent $\mathrm{EQE}$ can largely be attributed to the extremely low reflectance from $\mathrm{b}-\mathrm{Si}$, however, that alone does not explain the observed NIR responsivity with the used wafer thickness. To fully explain the NIR results, there needs to be increased optical path length due to light scattering from the $b$-Si on the front surface and/or reflection from the back surface metal. To quantify the effect of the increased optical path, we use an enhancement factor $(E n h)$ calculated with

$$
E n h=\frac{I Q E}{A}=\frac{E Q E /\left(1-R_{\text {front }}\right)}{1-\exp (-\alpha w)}
$$

where $A$ is absorption of light in a single pass through the wafer with thickness $w, R_{\text {front }}$ is the external reflectance of the front surface, and $\alpha$ is the absorption coefficient of light in silicon at a given wavelength. The enhancement factor should be limited to $\leq 2$ with a perpendicular light beam, even with a perfect backside mirror, when the front surface reflectance is zero ${ }^{2}$. However, equation 1 yields an enhancement factor of 3.6 at $1100 \mathrm{~nm}$, when using the absorption coefficient from ${ }^{9}$ and zero front surface reflectance. Hence, there needs to be scattering from the front or back surface. The light scattering from b-Si was further studied by comparing b-Si and planar induced junction photodiodes made from DSP FZ and epitaxial wafers in the next section.

\subsection{Planar vs. black silicon photodiodes}

The length of the optical path in silicon can also be used to describe the enhancement of light absorption ${ }^{10}$ using equation $I Q E=E Q E /\left(1-R_{\text {front }}\right)=1-\exp \left(-\alpha w_{\text {eff }}\right) \quad$, 
where $w_{\text {eff }}$ is the effective optical path length in silicon. Note that both Eq. 1 and 2 assume $100 \%$ internal charge collection efficiency for generated charge carriers which is a valid approximation based on Figure 1. Figure 2 shows the enhancement factors calculated from EQE measured from the b-Si and the corresponding planar photodiodes fabricated on $675 \mu \mathrm{m}$ thick DSP FZ wafers. The enhancement factors (and effective optical path lengths) are $3.0(2860 \mu \mathrm{m})$ and $2.1(1620 \mu \mathrm{m})$ for b$\mathrm{Si}$ and planar photodiodes at $1100 \mathrm{~nm}$, respectively. This suggests that there is significant scattering from b-Si which increases the light absorption up to $43 \%$ compared to planar photodiode.

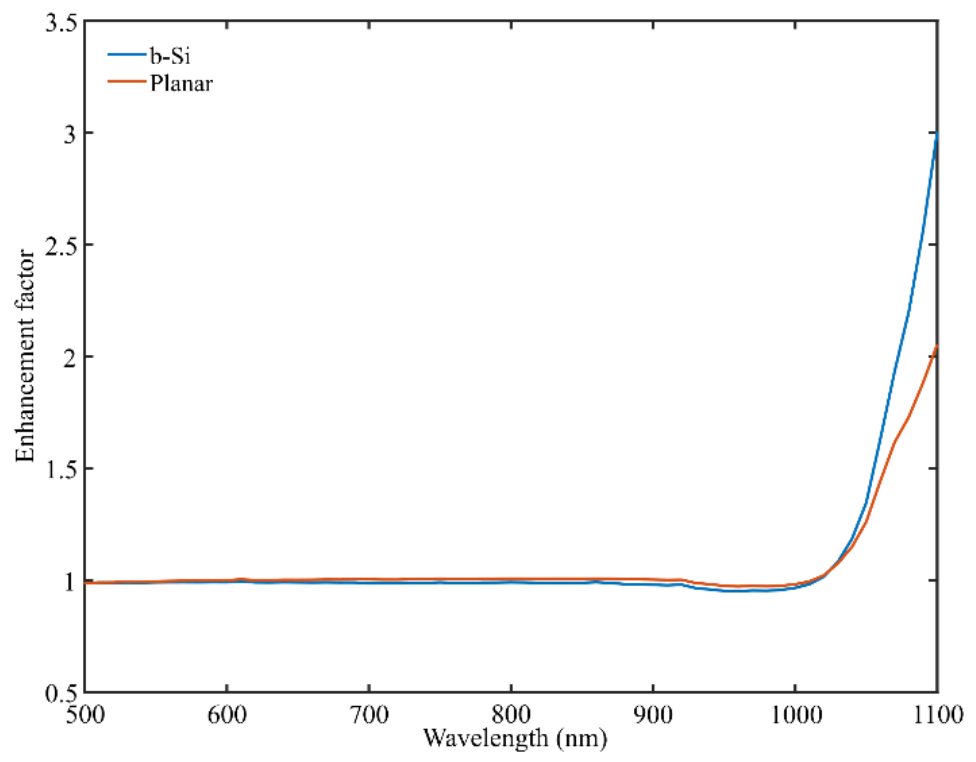

Figure 2. Enhancement factors calculated from EQE of planar and b-Si induced junction photodiodes made on identical DSP FZ wafers.

The penetration depth at wavelengths $\leq 1040 \mathrm{~nm}$ is short enough $(<450 \mu \mathrm{m})$ so that the reflectance from the back surface can be excluded when using the epitaxial wafers, making this substrate type ideal for studying only the effects arising from b-Si. Figure 3 shows the effective optical path lengths calculated for $b-S i$ and planar photodiodes fabricated on the epitaxial wafers. For comparison, the figure also includes a curve illustrating the ideal curve expected when $99 \%$ of the generated charge carriers are collected. At short wavelengths, all curves are overlapping, since all light is absorbed and collected in the epitaxial layer, as expected. However, both measured curves start to deviate from the ideal curve when the absorption depth exceeds the epitaxial layer thickness of $25 \mu \mathrm{m}$. The b-Si effective optical path length saturates to $40-42 \mu \mathrm{m}$ and planar to 34-35 $\mu \mathrm{m}$ before they start to increase again. The saturation point directly indicates the depth after which the photodiode collects no charge carriers. In an ideal epitaxial wafer, the planar photodiode saturation depth should match the epitaxial layer thickness but in reality, there is a finite minority carrier diffusion length also from the highly doped 
carrier. The EQE of this type of semi-infinite (i.e. no reflection from back surface gets collected) photodiode can be calculated from
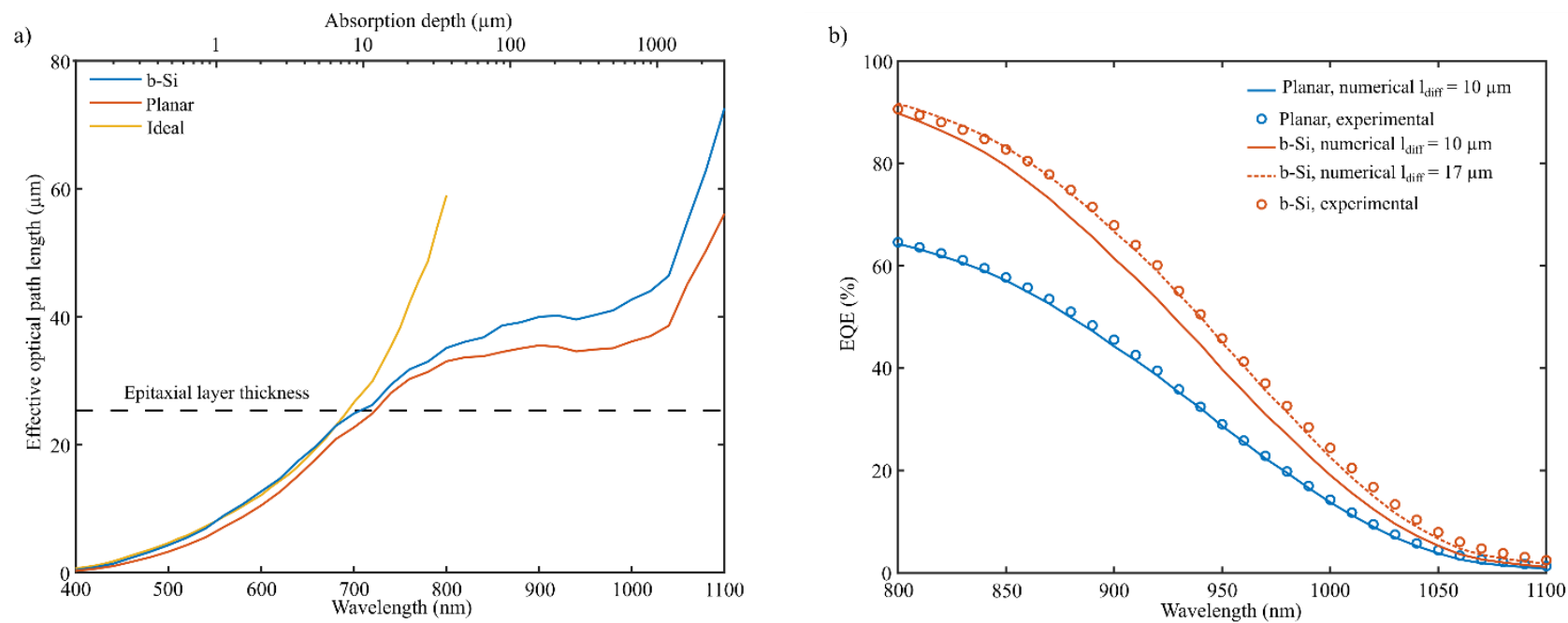

Figure 3. a) The effective optical length measured from planar and b-Si photodiodes made on epitaxial wafers together with comparison to ideal total absorption depth. b) Comparison of numerical and experimental EQE, when diffusion from the carrier below epitaxial layer is taken into account.

$$
E Q E=\left(1-R_{\text {front }}\right)\left(1-\frac{\exp \left(-\alpha W_{d e p}\right)}{\left.1+\alpha L_{d i f f}\right)}\right),
$$

where $W_{d e p}$ is the depletion layer thickness and $L_{d i f f}$ is the minority carrier diffusion length below the depletion layer ${ }^{11}$. Figure $3 \mathrm{~b}$ shows a comparison of calculated and measured EQE for $\mathrm{b}-\mathrm{Si}$ and planar photodiodes. The calculated curves assume a depletion layer of $25 \mu \mathrm{m}$ (i.e. 100\% charge collection from the epitaxial layer) and the diffusion length is obtained by fitting Equation 3 to the measured $\mathrm{EQE}$ curve. Best fits are obtained when $L_{\text {diff }}=10 \mu \mathrm{m}$ for the planar and $L_{\text {diff }}=17 \mu \mathrm{m}$ for the b-Si photodiode. Assuming bulk doping level of $4 \times 10^{18} \mathrm{~cm}^{-3}(\sim 0.01 \Omega \mathrm{cm})$ results in Auger recombination limited diffusion length of about $10 \mu \mathrm{m}^{12-13}$ which agrees well with the observation from the planar photodiode. The longer diffusion length of b-Si indicates that the effective optical path is increased by $7 \mu \mathrm{m}(20 \%)$ due to scattering. Both path lengths also match the saturation points seen in Figure 3a. Furthermore, an average light propagation angle of $33^{\circ}$ in b-Si can be estimated using the effective optical paths and basic trigonometry ${ }^{14}$. This value is in agreement with the simulation results in ${ }^{6}$. At wavelengths longer than $1050 \mathrm{~nm}$, the effective optical path length starts to increase again as the light reflected from the back surface starts to reach the epitaxial layer. 


\subsection{EQE at different incidence angles}

a)

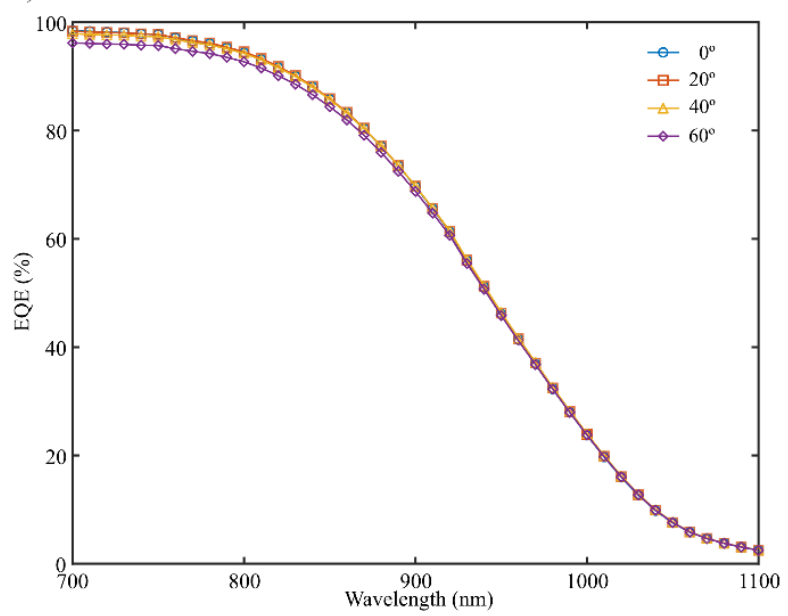

b)

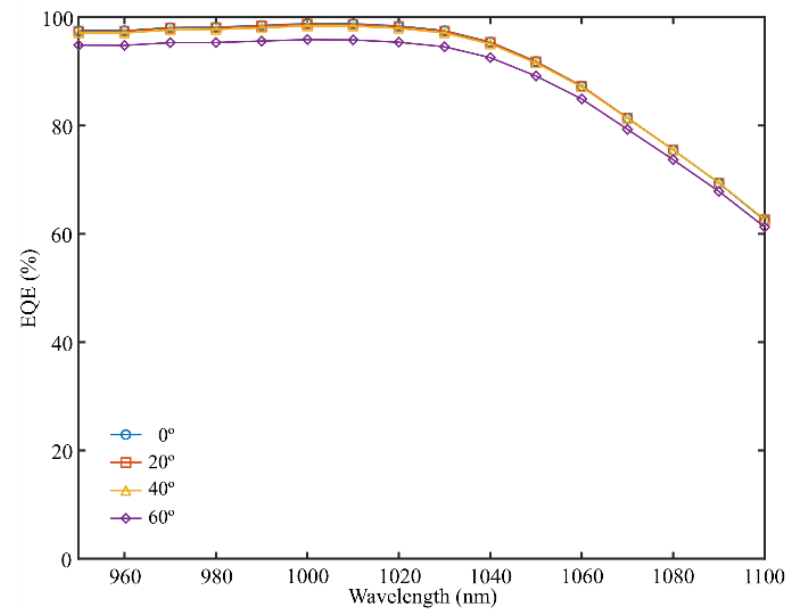

Figure 4. EQE of b-Si photodiodes made on a) epitaxial and b) DSP FZ wafers measured at different incidence angles between $0^{\circ}$ and $60^{\circ}$.

Figure 4 presents the EQE of b-Si photodiodes fabricated on epitaxial and DSP FZ wafers at different light beam incidence angles between $0^{\circ}$ and $60^{\circ}$. The curves illustrate that the high EQE (i.e. low reflectance) remains nearly constant even at $60^{\circ}$ for both wafer types, where EQE $>92 \%$ at visible wavelengths. Additionally, the results indicate that the difference in EQE is slightly smaller at longer wavelengths. Fitting Equation 3 to the curves in Figure 4a reveals that the effective optical path is $1 \mu \mathrm{m}$ longer at $60^{\circ}$ incidence angle compared to $0^{\circ}$. This corresponds to a $2^{\circ}$ change in the average light propagation angle suggesting that the scattering from b-Si has only a weak dependency on the incidence angle.

\section{CONCLUSIONS}

We have shown with certified measurements that the b-Si photodiodes exhibit even larger than 99\% EQE at visible light wavelengths. The EQE remains exceptionally high also at NIR wavelengths ( $84 \%$ at 1060 nm). Further investigations showed that in addition to low reflectance of $\mathrm{b}-\mathrm{Si}$, increased effective optical path length due to scattering has a major contribution to the enhancement seen at NIR. Comparison of identical photodiodes having b-Si and planar surface fabricated on thick DSP FZ wafers revealed that the scattering effect can increase the effective optical path length up to $43 \%$. This benefits especially devices such as backside illuminated photodiodes where thin substrates are essential. Further measurements from photodiodes fabricated on wafers having only thin a epitaxial active layer demonstrated that the effective light propagation angle due to scattering is $33^{\circ}$ when the light is perpendicular to the surface. Measurements with different incidence angles confirmed that the high EQE is maintained at angles up to $60^{\circ}$ and that the scattering effect has only a weak angle-dependency. Consequently, b-Si photodiode is an ideal solution also to applications where the light is not always perpendicular.

\section{ACKNOWLEDGMENTS}

We acknowledge the provision of facilities by Aalto University at OtaNano - Micronova Nanofabrication Centre. We acknowledge financial support from Business Finland. The authors from Aalto University are part of Academy of Finland Flagship Programme, Photonics Research and Innovation (PREIN). 


\section{REFERENCES}

[1] K. Yamamoto, A. Sakamoto, T. Nagano, and K. Fukumitsu, "NIR sensitivity enhancement by laser treatment for Si detectors," Nuclear Instruments and Methods in Physics Research, Section A: Accelerators, Spectrometers, Detectors and Associated Equipment, vol. 624, no. 2. pp. 520-523, 2010.

[2] L. Forbes, "Texturing, reflectivity, diffuse scattering and light trapping in silicon solar cells," Sol. Energy, vol. 86, no. 1, pp. 319-325, Jan. 2012.

[3] H. C. Yuan, V. E. Yost, M. R. Page, P. Stradins, D. L. Meier, and H. M. Branz, "Efficient black silicon solar cell with a density-graded nanoporous surface: Optical properties, performance limitations, and design rules," Appl. Phys. Lett., vol. 95, no. 12, 2009.

[4] M. A. Juntunen, J. Heinonen, V. Vähänissi, P. Repo, D. Valluru, and H. Savin, "Near-unity quantum efficiency of broadband black silicon photodiodes with an induced junction," Nat. Photonics, vol. 10, no. 12, pp. 777-781, 2016.

[5] M. Garin et al., "Black silicon UV photodiodes achieve $>130 \%$ external quantum efficiency," Jul. 2019, arXiv preprint arXiv:1907.13397, 2019.

[6] A. J. Bett et al., "Wave optical simulation of the light trapping properties of black silicon surface textures," Opt. Express, vol. 24, no. 6, p. A434, Mar. 2016.

[7] Filmetrics, Inc., "Reflectance calculator," Calculate Spectral Reflectance of Thin-Film Stacks, "No Title.” [Online]. Available: https://www.filmetrics.com/reflectance-calculator. [Accessed: 28-Aug-2019].

[8] J. Heinonen et al., "Black silicon n-type photodiodes with high response over wide spectral range," SPIE Opt., vol. 10231, p. 102310X, 2017.

[9] M. A. Green, "Self-consistent optical parameters of intrinsic silicon at $300 \mathrm{~K}$ including temperature coefficients," Sol. Energy Mater. Sol. Cells, vol. 92, no. 11, pp. 1305-1310, Nov. 2008.

[10] J. A. Rand and P. A. Basore, "Light-trapping silicon solar cells. Experimental results and analysis," in Conference Record of the IEEE Photovoltaic Specialists Conference, 1992, vol. 1, pp. 192-197.

[11] S. M. Sze and K. K. Ng, "Physics of Semiconductor Devices, 3rd Edition - Simon M. Sze, Kwok K. Ng," Phys. Semicond. Devices, 3rd Ed. John Wiley Sons, Inc.; NJ, pp. 164, 682, 2007.

[12] A. Richter, S. W. Glunz, F. Werner, J. Schmidt, and A. Cuevas, "Improved quantitative description of Auger recombination in crystalline silicon," Phys. Rev. B, vol. 86, p. 165202, 2012.

[13] D. B. M. Klaassen, "A unified mobility model for device simulation-II. Temperature dependence of carrier mobility and lifetime," Solid State Electron., vol. 35, no. 7, pp. 961-967, 1992.

[14] J. Geist, W. K. Gladden, and E. F. Zalewski, "Physics of photon-flux measurements with silicon photodiodes," J. Opt. Soc. Am., vol. 72, no. 8, pp. 1068-1075, 1982. 